\title{
A simple and sensitive fluorescence based biosensor for the determination of uric acid
} using $\mathrm{H}_{2} \mathrm{O}_{2}$-sensitive quantum dots/dual enzymes

\begin{abstract}
A novel optical detection system consisting of combination of uricase/HRP-CdS quantum dots (QDs) for the determination of uric acid in urine sample is described. The QDs was used as an indicator to reveal fluorescence property of the system resulting from enzymatic reaction of uricase and HRP (horseradish peroxidase), which is involved in oxidizing uric acid to allaintoin and hydrogen peroxide. The hydrogen peroxide produced was able to quench the QDs fluorescence, which was proportional to uric acid concentration. The system demonstrated sufficient activity of uricase and HRP at a ratio of 5U:5U and $\mathrm{pH}$ 7.0. The linearity of the system toward uric acid was in the concentration range of $125-1000 \mu \mathrm{M}$ with detection limit of $125 \mu \mathrm{M}$.
\end{abstract}

Keywords: Quantum dots; Enzyme; Uric acid; Fluorescence; Quenching 